\title{
Epidemic-related sites in covid-19 media reports
}

Bin Zhao ${ }^{1 *}$, Kuiyun Huang ${ }^{1}$ and Jinming $\mathrm{Cao}^{2}$

${ }^{1}$ School of Science, Hubei University of Technology, Wuhan, Hubei, China.

${ }^{2}$ School of Information and Mathematics, Yangtze University, Jingzhou, Hubei, China.

*Corresponding author: Bin Zhao, School of Science, Hubei University of Technology, Wuhan, Hubei, China Tel. /Fax: +86 1302851

7572. E-mail: zhaobin835@nwsuaf.edu.cn

Received date: June 06 2020; Accepted date: July 04, 2020; Published date: September 11, 2020

Citation: Zhao B., Huang K. and Cao J. (2020) Epidemic-related sites in covid-19 media reports; J. Clinical Research and Clinical

Trials. 2(3); DOI: 10.31579/2693-4779/005

Copyright: () 2020 Bin Zhao, This is an open-access article distributed under the terms of the Creative Commons Attribution License, which permits unrestricted use, distribution, and reproduction in any medium, provided the original author and source are credited.

\section{Abstract}

Background: Since the outbreak of the COVID-19 virus in Wuhan, China, in early 2020, the Chinese government has formed a mode of information disclosure. More than 400 cities have announced specific location information for newly diagnosed cases of novel coronavirus pneumonia, including residential areas or places of stay. We have established a conditional random field model and a rule-dependent model based on Chinese geographical name elements. Taking Guangdong Province as an example, the identification of named entities and the automatic extraction of epidemic-related sites are carried out. This method will help locate the spread of the epidemic, prevent and control the spread of the epidemic, and gain more time for vaccine clinical trials.

Methods: Based on the presentation form of the habitual place or place of stay of the diagnosed cases in the text of the web page, a conditional random field model is established, and a rule-dependent model is established according to the combination rule of the elements of the place words and the place name dictionary composed of provinces, cities and administrative regions.

Findings: The results of the analysis based on the conditional random field model and the rule-dependent model show that the location of confirmed cases of new coronavirus pneumonia in Guangdong Province in mid-February is mainly concentrated in Guangzhou, Shenzhen, Zhuhai and Shantou Cities. In Guangzhou, Futian District has more epidemicsites and Huangpu and Conghua District have fewer epidemic sites. Government officials in Guangzhou City should pay attention to Futian District.

Interpretation: Governments at all levels in Guangzhou Province have intervened to control the epidemic through various means in mid-February. According to the results of the model analysis, we believe that the administrative regions with more diagnosed locations should focus on and take measures such as blockades and control of personnel flow to control the disease in those administrative regions to avoid affecting other adjacent administrative regions.

Keywords: COVID-19; place name recognition; web crawler; part-of-speech tagging; conditional random field; elements of Chinese place name

\section{Introduction}

In early 2020, the COVID-19 virus began to erupt from Wuhan, Hubei, China, and it has only become a global epidemic in just a few months. In these months, China has turned into a crisis and the epidemic has been basically controlled, while other countries have moved towards the early days in some countries, the current situation is even worse than when the epidemic situation in China is the worst. One of the main reasons why China can overcome the epidemic is the openness and transparency of information from the central government to the local governments, and the release of relevant information on the official website on time every day.

From the epidemiological point of view, the specific location information such as the residential area or place of stay where the confirmed cases of new coronary pneumonia are published, is not only conducive to the individual in the life, but also to the government to establish an epidemic transmission channel to prevent and control the spread of the epidemic. In the webpages of these epidemic reports or media reports, the place of usual residence or place of stay of the confirmed cases is mostly expressed in various forms such as the body of the page, embedded text and screenshots on the page. In order to analyze the distribution of epidemicrelated locations from these sources of information in the past, manual search, extraction, and classification of specific information in the text have been carried out in the past, but the method requires a large amount of work, is inefficient, and lacks timeliness.

In recent years, as the technology of named entity recognition has gradually matured, this work has gradually shifted from manual extraction to automatic extraction, which not only reduces human and financial resources, but also speeds up the processing of tasks. Named entity recognition refers to identifying named entities in text and dividing them into corresponding entity types ${ }^{1}$. The general entity types include person names, place names, dates, and organization names. Because the Chinese characters are closely arranged in the Chinese text, the sentence is composed of multiple characters and there are no spaces between the words, so the difficulty of identifying named entities is increased. In order to improve the accuracy of recognition, named entity recognition technology from the initial rules and dictionary methods ${ }^{2}$ to the 
traditional statistical learning method ${ }^{3-5}$ to the current deep learning $\operatorname{method}^{6}$, for some common entities basically achieved higher accuracy.

\section{Methods}

\subsection{Data}

From the beginning of the outbreak of COVID-19, there will be news on the Internet every day to report the activities of newly diagnosed patients, so it provides us with original unstructured data for collecting diagnosis locations. We collect official notifications and media reports on the itinerary of the activities of the diagnosed patients associated with
Guangdong Province from January 29, 2020 to February 19, 2020. The data comes from 366 webpages in 152 media. The original data is obtained through web crawler technology, and the data includes the main content of the webpage, the media of the news release and the corresponding URL. The basic principle of a web crawler is to simulate a browser making HTTP requests. The crawler client sends a request to the web server through the HTTP request, downloads the web page after obtaining the corresponding server, and completes the crawling work of the crawler system ${ }^{7}$. Part of the data is shown in Table 1.

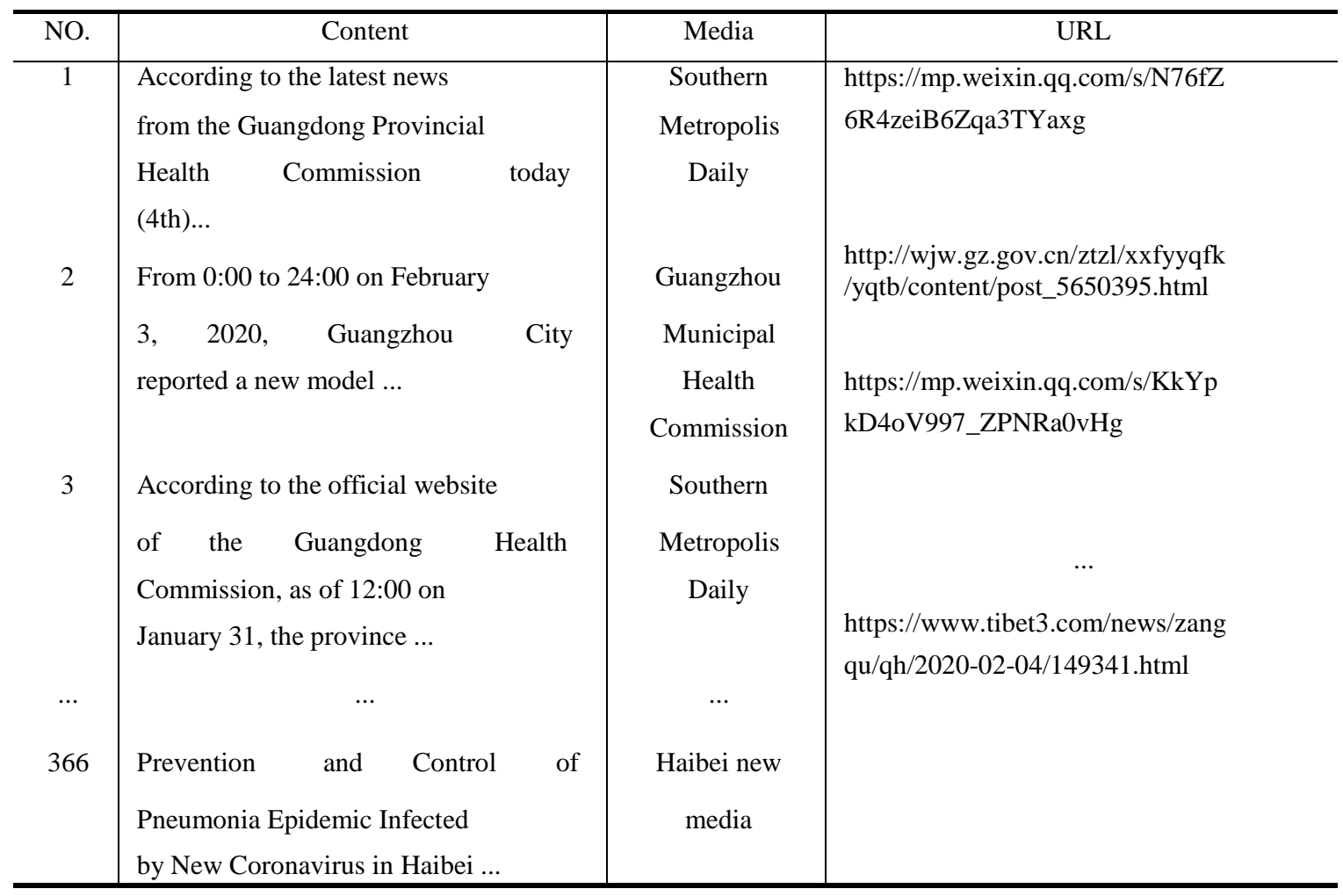

Table 1: Part of the diagnosis locations data

\subsection{Text data preprocessing}

The preprocessing of text data means that before performing named entity recognition, we must first determine whether there are missing values in the data. After checking, there are no missing values, and then we need to convert the data into a format that the model can easily handle. For example: delete some unnecessary character strings, split each press release in sentence units and remove the same sentence, etc. The part-of-speech feature is used in Peking University's14.

\subsection{The model}

Based on the collected unstructured text data, we tried to identify and extract the place words in the text through named entity recognition technology, and then classify the recognized place words according to a certain rule and divide them into provinces, cities, and administrative regions. As well as the four major categories of specific locations, the final analysis of the extracted location information will provide key evidence for the subsequent construction of the epidemic development model and the assessment and prediction of the source of infection, transmission speed, transmission path, and transmission risk.

There are three main methods for studying Chinese place name recognition: rule-based methods, statistics-based methods, and deep learning-based methods. The rule-based method is intuitive and natural, and is easy to understand and expand by humans. However, rule writing depends on specific language knowledge and domain knowledge. The rules are more complicated, it is difficult to cover all the modes, and the portability is also poor ${ }^{8}, 9$. Statistics-based methods do not require excessive language knowledge and domain knowledge and are highly portable, but require manual annotation of the corpus and selection of appropriate statistical learning models and parameters ${ }^{10}$ 11, 12 . Deep learning-based methods do not require overly complex feature engineering, and can automatically discover information from the input to form an end-to-end model ${ }^{13}$. Considering that the collected text data is limited by time and data, the content of this paper is mainly based on the first two methods.

Recently, named entity recognition has achieved good results in some limited entity types. For example, the recognition effect on the names of 
people, places and organizations in news corpus is remarkable. Chinese place name recognition can be regarded as a sequence labeling problem. The place name is a combination of multiple words arranged in a certain order, and the place name entity recognition is the combination of marking the correct names from these word sequences. The conditional random field model combines the advantages of the maximum entropy model and the hidden Markov model, and can be used for the labeling and segmentation of sequence data $^{5}$. Therefore, the effective solution to the sequence labeling problem is the conditional random field model ${ }^{14}$. We chose the conditional random field model as the identification model for epidemic locations.

\subsection{Maximum likelihood method for estimation}

Suppose there is a set of sample sets $D=\left\{x^{j}, y^{j}\right\}, \forall j=1,2,7, N$ for the training data, where the samples are Independent of each other, and $p(x, y)$ is the empirical probability of $(x, y)$ in the training samples. For a certain conditional model $p(y \mid x, \theta)$, the maximum likelihood function

Formula of the training data sample set can be expressed as:

$$
L(\theta)=\prod p(y \mid x, \theta) \stackrel{\sim}{p}(x, y)
$$

Take the logarithmic form:

$$
L(\theta)=\prod \tilde{p}(x, y) \log p(y \mid x, \theta
$$

Since we are studying a conditional random field model, its conditional probability can be expressed as: $p(y \mid x, \theta)=\frac{1}{Z(x)} \exp \left(\sum_{i=1}^{n+1} \sum_{k} \lambda_{k} f_{k}\left(y_{i-1}, y_{i}, x\right)+\sum_{i=1}^{n} \sum_{k} \mu_{k} g_{k}\left(y_{i}, x\right)\right)$

Where $\lambda$ and $\mu$ are the parameters to be estimated, and $f$ and $g$ are the vectors of theEigen functions differentiate the

$$
\frac{\partial L(\theta)}{\partial \lambda_{k}}=\sum_{x, y} \tilde{p}(x, y) \sum_{i=1}^{n+1} f_{k}\left(y_{i-1}, y_{i}, x\right)-\sum_{x, y} \tilde{p}(y \mid x, \theta) \sum_{i=1}^{n+1} f_{k}\left(y_{i-1}, y_{i}, x\right)
$$

parameter $\lambda k$ :

Let the value of the above formula be 0 to meet some of the constraints and find $\mid k$. In the same way, $k$ can also be obtained, so that || can be parsed, but this method may not always be able to directly find the solution value.

\subsection{Feature selection for Chinese location recognition}

The training set used in this article is the annotated corpus of the People's Daily in January 1998. It uses the part-of-speech annotation set of Peking University. All the names of people, places, and organizations have been marked. Word segmentation sentences, and in order to make the features of entity extraction more obvious, these coarse word segmentation sentences need to be finely segmented.

The 5n-gram template can fully express the lexeme information of the word in the word. Among them B (the beginning word of the named entity), $M$ (the middle word of the named entity), $E$ (the tail of the named entity), $\mathrm{S}$ (the single word constitutes the named entity), $\mathrm{N}$ (unnamed entity). The three types and combinations of entities form various labels. The template can effectively mark the position of the word in the word, so that the system can use the position feature to identify the boundary of the word. Table 2 shows the labeled labels

\begin{tabular}{c|c}
\hline Output tags & Explanations for different tags \\
\hline Per-B & The start word(s) of the name entity \\
\hline Per-M & The middle word(s) of name entities \\
\hline Per-E & The ending word(s) of name entity \\
\hline Per-S & The individual word(s) constitute name entity \\
\hline Loc-B & The start word(s) of place name entity \\
\hline Loc-M & The middle word(s) of place name entity \\
\hline Loc-E & The ending word(s) of place name entity \\
\hline Loc-S & The individual word(s) constitute a place name entity \\
\hline Org-B & Start word(s) of organization name entity \\
\hline Org-M & The middle word(s) of organization name entity \\
\hline Org-E & The ending word(s) of organization name entity \\
\hline Org-S & The individual word(s) constitute organization name entity \\
\hline $\mathrm{N}$ & Unnamed entity word \\
\hline
\end{tabular}

\subsection{Feature template selection}

The conditional random field is very dependent on the selection of features, which has a great influence on the accuracy of the final recognition. Theoretically, if more context information is collected around the current word, that is, the larger the value of the observation window, the richer the information obtained and the more accurate the judgment of the current word.

But once the observation window is too large, the calculation of too much 
information will make the model identification efficiency inefficient and affect the operation efficiency. If the value of the window is too small, the relevant dependency information cannot be fully utilized, affecting the accuracy of recognition. So choosing the right window size is the premise of choosing the right feature template. The window size selected in this article is 2 .

The base feature is the most basic feature that is stronger than the character itself, including the current character or key, the position of the first character in the pre-word and part of speech. Part-of-speech features can often improve the degree of discrimination.

For example, named entities are often noun part-of-speech words, while verb part-of-speech words are rarely used as named entities. For a wordbased entity tagging system, the part-of-speech feature is to use the partof-speech of the word in which the word is located. Based on these, we establish the basic features of the template as shown in Table 3.

\begin{tabular}{|c|c|c|}
\hline Transfer features & Word features & Part of speech features \\
\hline \multirow[t]{4}{*}{$y_{t}$} & \begin{tabular}{l|l} 
word $_{t}$ & $\mid 2$ \\
word $_{t}$ & $\mid 1$
\end{tabular} & \begin{tabular}{c|c}
$\operatorname{tag}_{t}$ & $\mid 2$ \\
$\operatorname{tag}_{t}|| 1$
\end{tabular} \\
\hline & word $_{t}$ & $\operatorname{tag}_{t}$ \\
\hline & word $_{t} \mid 1$ & $\operatorname{tag}_{t} \backslash 1$ \\
\hline & word $_{t} \mid 2$ & $\operatorname{tag}_{t \quad 2}$ \\
\hline
\end{tabular}

Table 3: The basic features of the template

Where $t$ represents the position where the feature is currently being extracted, y represents the label, word represents the word, and tag represents the part of speech. Considering that there are so many ways to combine words, there are not as many binary grammatical features as part of speech

\subsection{Entity relationships}

The seven types of relationships between entities are: partial overall relationship, geographical location relationship, generic relationship, metaphor relationship, manufacturing use relationship, organizational structure dependency relationship, and character relationship.

For this work, what is studied is the relationship between locations in an outbreak press release, so more attention is paid to the identification of geographic location relationships. According to the characteristics of the recognized text itself, the geographical location relationship is classified in detail (Table 4):

\begin{tabular}{|c|c|c|c|}
\hline \multicolumn{2}{|c|}{ Category } & Symbol & Word \\
\hline & Province & S1 & $\begin{array}{l}\text { Provinces, Municipalities, Autonomous regions, } \\
\text { Special administrative regions }\end{array}$ \\
\hline Administrative & City & $\mathrm{S} 2$ & $\begin{array}{c}\text { City, Region, League, Autonomous prefecture } \\
\text { County, Flag, Qu, District }\end{array}$ \\
\hline & County & S3 & \\
\hline & Town & S4 & Township, Town, Street office \\
\hline & Village & S5 & Village, Zhuang, Tun, Li \\
\hline Street & & S6 & Road, Avenue, Road, Street, Lane, Alley, Strip \\
\hline $\begin{array}{l}\text { Residential } \\
\text { area }\end{array}$ & & S7 & Garden, Square, Residence, Apartment \\
\hline Landmarks & & S8 & $\begin{array}{l}\text { Building, Square, Hotel, Center, Building, Floor, } \\
\text { Field, Square, Pavilion, Residence }\end{array}$ \\
\hline House number & & S9 & NO., \# \\
\hline
\end{tabular}


The geographical location relationships that may be involved in the entry are divided into four categories: provinces, cities, administrative regions and geographical locations. The head word of a relationship can be not only a noun but also a verb. The system needs to classify the identified entity relationships. The specific division is shown in Table 5.

Table 5: Specific geographical name division

\begin{tabular}{c|c}
\hline Category & Symbol \\
\hline province & $\mathrm{S} 1$ \\
city & $\mathrm{S} 2$ \\
administrative region & $\mathrm{S} 3$ \\
geographical location & $\mathrm{S} 4, \mathrm{~S} 5, \mathrm{~S} 6, \mathrm{~S} 7, \mathrm{~S} 8, \mathrm{~S} 9$ \\
\hline
\end{tabular}

\subsection{Entity relationships extraction method}

Recognition of relational semantics is constantly evolving at any time and is divided into methods based on rule matching and methods based on machine learning. The method based on rule template matching is to define the rule template beforehand, and compare the statement with the rule template during the relationship identification. If the statement matches the characteristics of the characteristic template, it means that the entity in the statement has the relationship specified in the template Attributes. The disadvantage is that it requires more professional linguists to write a large number of feature templates, which takes a long time and has poor portability.

The method based on machine learning is a method that uses various pattern recognition feature models to calculate the entity relationship features and weight values in sentences through related algorithms. There are currently two popular types of machine learning methods for dealing with entity relationships, namely kernel-based methods and feature vector-based methods.

The purpose of our research is to perform location extraction. The feature template of geographic location relationship is relatively fixed and the portability is high. Therefore, we will use the rule-based matching method to extract the identified place words for relationship. There are three aspects of corpus preprocessing, rulemaking.

\subsection{Corpus preprocessing}

The preprocessing of corpus is mainly through the steps of word segmentation and entity recognition, which transform the sentences in the corpus into a stream of words with entity identification. Since entity relationship extraction is a relationship between two entities, the sentences with less than two place name entities in the text are filtered out, and the sentences containing two or more place name entities are used as recognition corpus.

\subsection{Gazetteer}

There may not be a complete place name in some sentences, for example: only the information of the administrative region, and no information of the province or city. At this time, we need to collect all the names of provinces, cities and districts in China and establish a dictionary framework. Part of the data is shown in Figure 1.

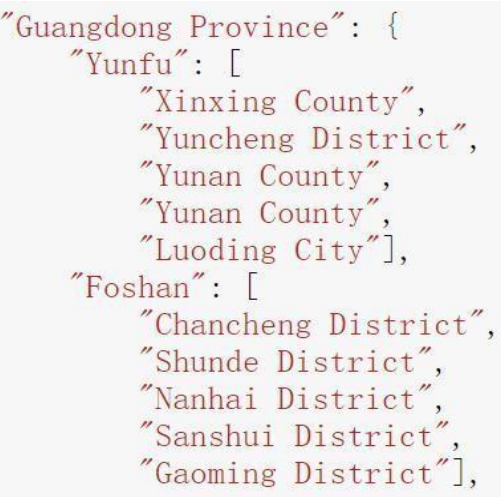

Figure 1: Structural framework of some Chinese provinces, cities and administrative regions

Since the object of our research is Guangzhou, China, we will not consider the COVID-19 epidemic situation of abroad. Therefore, we need to collect major foreign countries and place names from the Internet and establish a dictionary framework. Part of the data is shown in Figure 2.

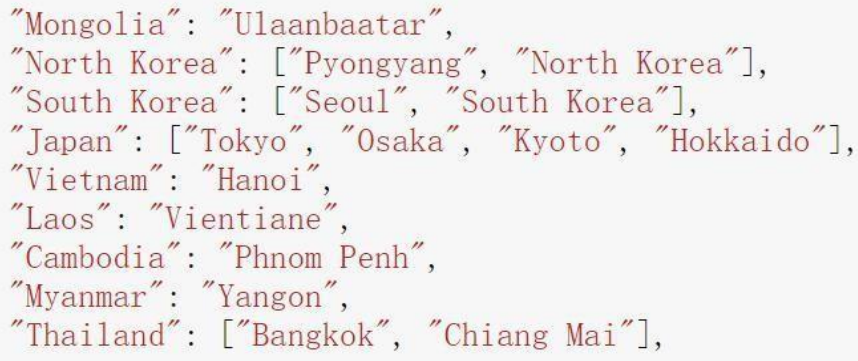

Figure 2: Structural framework of some foreign countries place name

\subsection{Rule making}

By studying the structural characteristics of epidemic location words, establishing rules based on regular expressions to extract word-by-word the location words identified by the named entity recognition, and then putting the extracted relevant location information into an appropriate data structure for subsequent deal with.

By analyzing location words, a total of 20 matching rules were established for 4 categories of location information. Each rule is a regular expression composed of keyword(s) of which there are 9 categories of keyword(s). The rule form is as follows:

Rule 1: (*?) (S1)?

$\mathrm{S} 1=$ Provinces $\mid$ Municipalities $\mid$ Autonomous regions $\mid$ Special administrative regions Rule 2: (.*?) (S2)?

S2 = City $\mid$ Region, League $\mid$ Autonomous prefecture Rule 3: (.*?) (S3)

S3 = County $\mid$ Flag $\mid$ Qu $\mid$ District Rule 4: (.*?) (S4)

S4 = Township $\mid$ Town $\mid$ Street office Rule 5: (.*?) (S5)

S5 = Village $\mid$ Zhuang $\mid$ Tun, Li Rule 6: (.*?) (S6)

S6 = Road $\mid$ Avenue $\mid$ Road $\mid$ Street $\mid$ Lane $\mid$ Alley $\mid$ Strip Rule 7: (.*?) (S7)

S7 = Garden $\mid$ Square $\mid$ Residence $\mid$ Apartment Rule 8: (.*?) (S8) 
S8 = Building $\mid$ Square $\mid$ Hotel $\mid$ Center $\mid$ Building | Floor $\mid$ Field $\mid$ Square | Pavilion | Residence Rule 9: (.*?) (S9)

S9 = NO. $\mid \#$

Rule 10: "Province" = Rule 1

Rule 11: "City"= Rule 2

Rule 12: "administrative region" = Rule 3

Rule 13: "geographical location" = [Rule 4, Rule 5, Rule 6, Rule 7, Rule 8 , Rule 9]

Rule 14: Delete words that meet the Rule 1 to Rule 9 conditions, but do not belong to any place names. For example: "Epidemic Area", "Common Youth City", "Community", "Outer Province", "Inner Province", "You Province", "Reprinted City", "Ministry of Labor", etc.

Rule 15: Delete words that are incorrectly marked in the recognition of named entities. For example: "Sputum", "when getting on the train", "from the day", "and", "more", etc.

Rule 16: Delete words that belong to place names but are not related to this study. For example: "People's Republic of China", "China", "People's Hospital", "Chest Hospital", etc.

Rule 17: Use foreign gazetteer to delete foreign place names

Rule 17: If "administrative region" != "“" and "City" == "”, find the corresponding place name from the Chinese gazetteer and fill it in "City"

Rule 18: If "administrative region" != "" and "Province" == "”, find the corresponding place name from the Gazetteer and fill it in "Province"

Rule 19: If "City" != "” and "Province" == "”, find the corresponding place name from the Gazetteer and fill it in

"Province"

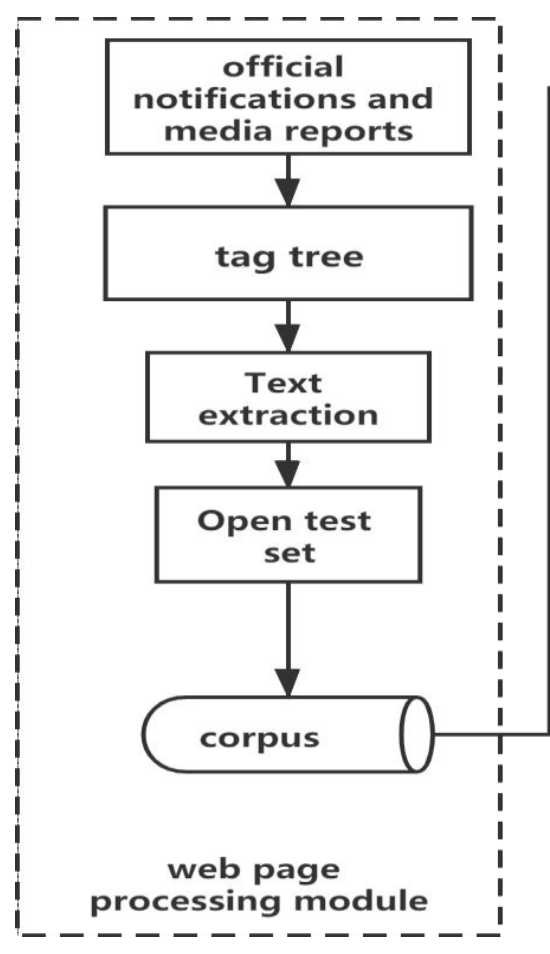

Rule 20: According to the principle of proximity, re-sort "administrative region" and "geographical location", and stitch the sorted words together.

$$
\begin{gathered}
P=\frac{\text { Correctly identify the number of entities in this category }}{\text { Total number of entities identifying this category }} \\
R=\frac{\text { Correctly identify the number of entities in this category }}{\text { Total number of named entities of this category }} \\
\qquad F_{1}=\frac{2 \times P \times R}{P+R}
\end{gathered}
$$

For example: after processing "Bailudong Street / Shili Bay / Suxian District", it becomes "Shili Bay, Bailudong Street, Suxian District"

\subsection{Model evaluation criteria}

For the evaluation of the model, the F1-score evaluation index is used for evaluation. For each type of named entity and relationship extraction, these three indicators are defined: for the evaluation of the model, the F1score evaluation index is used for evaluation. For each type of named entity and relationship extraction, these three indicators are defined:

\subsection{Overall framework for automatic extraction of} Chinese place names

The purpose of this article is to process the text information in the web page until the entities and relationships are displayed. The implementation process is mainly divided into two modules: web page processing module and entity and relationship recognition module. The frame diagram of the automatic extraction of Chinese locations is shown in Figure 3.

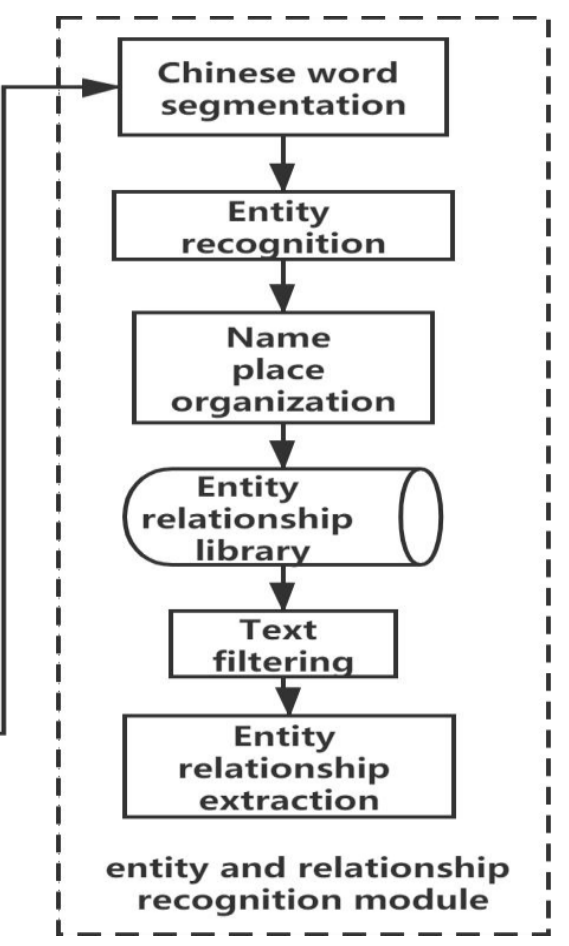




\section{Results}

\subsection{Place name entity recognition result}

This article uses the corpus marked by People's Daily in January 1998, of which $80 \%$ is selected as the training set, the remaining $20 \%$ is used as the closed test set, and the COVID-19 outbreak news release crawled through the Internet will be used as the open test set. The results of entity recognition are shown in Table 6.

\begin{tabular}{c|c|c|c|c|c|c|c}
\hline \multicolumn{4}{c|}{ Closed test corpus } & \multicolumn{3}{c}{ Open test corpus } \\
\hline Entity type & $\mathrm{P}$ & $\mathrm{R}$ & $\mathrm{F}$ & Entity type & $\mathrm{P}$ & $\mathrm{R}$ & $\mathrm{F}$ \\
\hline Place entity & 0.854 & 0.831 & 0.870 & Place entity & 0.781 & 0.763 & 0.771 \\
Loc-B & 0.881 & 0.852 & 0.872 & Loc-B & 0.732 & 0.718 & 0.720 \\
Loc-M & 0.873 & 0.852 & 0.870 & Loc-M & 0.729 & 0.706 & 0.711 \\
Loc-E & 0.869 & 0.841 & 0.857 & Loc-E & 0.703 & 0.690 & 0.692 \\
Loc-S & 0.896 & 0.853 & 0.878 & Loc-S & 0.693 & 0.752 & 0.764 \\
\hline
\end{tabular}

It can be seen from the experimental data that the results of place name recognition have higher accuracy. The closed training set can reach the $\mathrm{F}$ value of 0.870 , and the open training set can reach the $F$ value of 0.771 . As can be seen from the results of entity recognition, there are mainly the following types of incorrect recognition of place entities:

a) There are abbreviations for cities and provinces in the text, and it is possible to identify place names in ambiguous forms. For example: "Zhongshan" can be either a city in Guangdong Province or an administrative district in Dalian, Liaoning Province;

b) Some place names appear in multiple cities. For example: "Baojian
Road" is the road name of many cities. When there are multiple cities in one sentence, it is not easy to determine which city this road name belongsto;

c) Place words in different places have different meanings. For example: The "Bajiao Tower" can be the name of a building or a town;

d) The wrong labeling of the entity label itself leads to the wrong words in the recognition location. For example: The words "Sputum", "when getting on the train", "from the day", "and", "more" are not place names, but they are classified as place names according to the algorithm.

\subsection{Place entity extraction results}

\begin{tabular}{c|c|c|c}
\hline Category & P & R & F \\
\hline Province & 0.776 & 0.741 & 0.765 \\
City & 0.753 & 0.712 & 0.682 \\
Administrative region & 0.702 & 0.653 & 0.501 \\
\hline
\end{tabular}

Table 7: Place entity relationship recognition result

The location extraction link is very dependent on the central indicator. In the case of other features that are the same, different types of central words will make the entity relationship pairs form different semantic relationships. Therefore, the central indicator thesaurus needs to identify the wrong entity center. The center of is extracted and added to the central indicator thesaurus to increase the recognized features. The maintypes of errors in entity relationship Extraction are:

a) Some place name words omit entity words or substitute pronouns. For example: many provinces and cities have omitted the entity words "Province" or "City"; b) Two identical entities in the text have different relationship classifications and cannot determine priority. For example: "Jilin" is both a province name and a city name;

c) Multiple locations are involved in one sentence. There are multiple place words in a sentence, it is not easy to judge when recognizing the relationship, and the subordinate relationship can only be judged based on the position of the word in the sentence

\subsection{Final results}

As some provinces in the form are not filled in, the locations of 
Guangdong Province are selected and sorted according to the information $\quad$ in the city column. The results are shown in Table 8.

\begin{tabular}{|c|c|c|c|c|c|}
\hline City & $\begin{array}{l}\text { Administrative } \\
\text { region }\end{array}$ & $\begin{array}{l}\text { The number of } \\
\text { geographical } \\
\text { location }\end{array}$ & City & $\begin{array}{c}\text { Administrative } \\
\text { region }\end{array}$ & $\begin{array}{c}\text { The number of } \\
\text { geographical } \\
\text { location }\end{array}$ \\
\hline \multirow[t]{7}{*}{ Guangzhou } & Yuexiu & 11 & \multirow[t]{5}{*}{ Shenzhen } & Futian & 19 \\
\hline & Tianhe & 10 & & Luohu & 18 \\
\hline & Qiewan & 10 & & Longgang & 16 \\
\hline & Haizhu & 7 & & Yantian & 2 \\
\hline & Fanyu & 1 & & Baoao & 1 \\
\hline & Huangpu & Unknown & \multirow[t]{4}{*}{ Huizhou } & Huidong & 5 \\
\hline & Conghua & Unknown & & Boluo & 4 \\
\hline \multirow[t]{4}{*}{ Foshan } & Shunde & 4 & & Huicheng & 1 \\
\hline & Nanhai & 3 & & Huiyang & Unknown \\
\hline & Chancheng & 2 & \multirow[t]{3}{*}{ Zhuhai } & Xiangzhou & 15 \\
\hline & Sanshui & Unknown & & Jinwan & 1 \\
\hline \multirow[t]{7}{*}{ Meizhou } & Meijiang & 2 & & Doumen & Unknown \\
\hline & Meixian & Unknown & \multirow[t]{4}{*}{ Jiangmen } & Pengjiang & 3 \\
\hline & Pingyuan & Unknown & & Jianghai & 1 \\
\hline & Dapu & Unknown & & Heshan & 1 \\
\hline & Fengshun & Unknown & & Xinhui & Unknown \\
\hline & Jiaoling & Unknown & \multirow[t]{2}{*}{ Jieyang } & Puning & Unknown \\
\hline & Wuhua & Unknown & & Jiexi & Unknown \\
\hline
\end{tabular}

\begin{tabular}{|c|c|c|c|c|c|}
\hline Maoming & Binhai & Unknown & Qingyua & Qingcheng & Qingxin \\
& Dianbai & Unknown & $\mathrm{n}$ & Unknown \\
& Huazhou & Unknown & & Yangshan & Unknown \\
& Xinyi & Unknown & & Yingde & Unknown \\
\hline Shantou & Chaoyang & 16 & & Lianshan & Unknown \\
\hline
\end{tabular}




\begin{tabular}{|c|c|c|c|c|c|}
\hline & $\begin{array}{l}\text { Nan'ao } \\
\text { Longhu }\end{array}$ & $\begin{array}{l}2 \\
1\end{array}$ & Shanwei & $\begin{array}{l}\text { Luhe } \\
\text { Lufeng }\end{array}$ & $\begin{array}{l}1 \\
1\end{array}$ \\
\hline & $\begin{array}{l}\text { Jingping } \\
\text { Chaonan }\end{array}$ & $\begin{array}{c}1 \\
\text { Unknown }\end{array}$ & $\begin{array}{c}\text { Yangjian } \\
\mathrm{g}\end{array}$ & $\begin{array}{c}\text { Yangxi } \\
\text { Yangchun }\end{array}$ & $\begin{array}{c}1 \\
\text { Unknown }\end{array}$ \\
\hline & Chenghai & Unknown & & Yangdong & Unknown \\
\hline \multirow[t]{7}{*}{ Zhanjiang } & Potou & 2 & & Jiangcheng & Unknown \\
\hline & Xiashan & 2 & \multirow[t]{5}{*}{ Zhaoqing } & Guangning & Unknown \\
\hline & Chikan & 1 & & Dinghu & Unknown \\
\hline & Lianjiang & 1 & & Duanzhou & Unknown \\
\hline & Wuchuan & Unknown & & Gaoyao & Unknown \\
\hline & Leizhou & Unknown & & Sihui & Unknown \\
\hline & $\begin{array}{c}\text { Development } \\
\text { zone }\end{array}$ & Unknown & \multirow[t]{2}{*}{$\begin{array}{c}\text { Zhongsh } \\
\text { an }\end{array}$} & $\begin{array}{c}\text { Development } \\
\text { zone }\end{array}$ & Unknown \\
\hline Heyuan & Yuancheng & 1 & & Shiqi & 1 \\
\hline
\end{tabular}

Table 8: Epidemic sites in Guangdong Province

* Unknown: The cities and administrative regions have appeared in the list, but there is no specific location

It can be inferred from Table 7 that the cities of the COVID-9 epidemic area in Guangdong Province are mainly concentrated in Guangzhou, Shenzhen, Zhuhai and Shantou. Among them, the epidemic areas in Guangzhou are concentrated in Yuexiu, Tianhe and Qiewan, the epidemic areas in Shenzhen are in Futian, Luohu and Longgang, the epidemic areas in Zhuhai are in Xiangzhou, and the epidemic areas in Shantou are in Chaoyang. Guangzhou has the widest epidemic area, the epidemic area of Heyuan is relatively small.

\section{Discussion}

There is no doubt that there are many aspects that reflect the severity of COVID-19 in an area. Considering the spread of the epidemic, the number of locations where the epidemic has occurred is one aspect. However, considering that COVID-19 is mainly concentrated in one place, it is concentrated, and the infection is mainly close-range infection. If considering the spread of the epidemic, it should be considered from the early stage of the COVID-19 infectious disease. However, due to the long incubation period of COVID-19 pneumonia, the infection may have spread before a case shows symptoms. However, this method can be used to find the source of the disease, so as to target areas that need attention.

\section{Limitations}

Although the conditional random field model is used in this paper to realize the entity recognition of the epidemic location, the rule-dependent model is used to extract the entity relationship of the COVID-19 pneumonia patient's itinerary. But the following aspects still need to be improved:

a) The conditional random field model can rely on the information in this article for a long distance to improve the recognition accuracy, but this also increases the cost of the model and makes the recognition efficiency inefficient. The conditional random field model should be appropriately improved in the future, so that the efficiency can be improved while ensuring accuracy.

b) In the process of entity recognition and relationship extraction, the entity pairs are identified in sentence units, so that once two related entity pairs exist in two sentences, or after a complex pronoun is used in the sentence it will cause mistakes in relationship extraction. In the future, we should need to increase the research on the pronoun entity.

\section{Conflict of interest}

We have no conflict of interests to disclose and the manuscript has been read and approved by all named authors.

\section{Acknowledgement}

This work was supported by the Philosophical and Social Sciences Research Project of Hubei Education Department (19Y049), and the Staring Research Foundation for the Ph.D. of Hubei University of Technology (BSQD2019054), Hubei Province, China.

\section{References}

1. Chen S D, Ouyang X Y. (2020) Overview of Named Entity Recognition Technology [J/OL]. Radio Communications Technology: 1-11

2. Rau L F. (1991) Extracting Company Names from Text[C]// Proceedings of the Seventh IEEE Conference on Artificial Intelligence Application. IEEE, 1: 29-32.

3. Rathaparkhi A. (1996) A Maximum Entropy Model for Part-of- 
speech Tagging[C]// Conference on Empirical Methods in Natural Language Processing, 133-142.

4. Mccallum A, Freitag D, Pereta F C N. (2000) Maximum Entropy Markov Models for Information Extraction and Segmentation[C]// Icml, 17: 591-598.

5. Lafferty J, Mccallum A, Pereira F C N. (2001) Conditional Random Fields: Probabilistic Models for Segmenting and Labeling Sequence Data[C]// Proceedings of the $18^{\text {th }}$ International Conference on Machine Learning (ICML 2001): 282-289.

6. Collobert R, Weston J, Bottou L, et al. (2011) Natural Language Proceeding (almost) from Scratch [J]. Journal of Machine Learning Research, 12(8): 2493-2537.

7. Pan X Y, Chen L, Yu H M, et al. (2020) Survey on Research of Topic Crawling Technique [J]. Application Research of Computers, 37(4): 961-965+972.

8. Tan K K. (2011) Rule-based Chinese Address Segmentation and Matching Methods [D]. Qingdao: Shandong University of Science and Technology.

9. Du P, Liu Y (2011) Recognition of Chinese Place Names Based on Ontology [J]. Journal of Northwest Normal University (Natural Science), 47(6): 87-93.

10. Qiu S, A Y, Wang F Y, et al. (2011) Study on Automatic Recognition of Chinese Location Names Based on Statistical Method [J]. Computer Technology and Development, 21(11): 35-38.

11. Tang X R, Chen X H, Zhang X Y. (2010) Research on Toponym Resolution in Chinese Text [J]. Geomatics and Information Science of Wuhan University, 35(8): 930-935.

12. Aaron L F H, Derek F W, Lidia S C. (2013) Chinese Named Entity Recognition with Conditional Random Fields in the Light of Chinese Characteristics[M]. LP\&IIS2013, Warsaw: Springer,

13. Wei Y, Li H F, Hu D L, et al. (2018) A Method of Chinese Place Name Recognition Based on Composite Features [J]. Geomatics and Information Science of Wuhan University, 43(1): 17-23.

14. Yu S W, Duan H M, Zhu X F, et al. (2020) The Basic Processing of Contemporary Chinese Corpus at Peking University Specification [J]. Journal of Chinese Information Processing, (5): 49-64.
This work is licensed under Creative Commons Attribution 4.0 License

To Submit Your Article Click Here: Submit Article

DOI: $10.31579 /$ CRCT.2020/005
Ready to submit your research? Choose Auctores and benefit from:

* fast, convenient online submission

* rigorous peer review by experienced research in your field

* rapid publication on acceptance

* authors retain copyrights

* unique DOI for all articles

* immediate, unrestricted online access At

Auctores, research is always in progress.

Learn more www.auctoresonline.org/journals/clinical-research-andclinical-trials 